\title{
Adsorption of Polymer Mixtures from Diluted and Semidiluted Solutions
}

\author{
Yuri S. Lipatov \\ Institute of Macromolecular Chemistry, \\ Ukrainian Academy of Sciences, \\ 48 Kharkov Shaussee, Kiev, 252160, USSR \\ (Received February 8, 1991)
}

\begin{abstract}
The adsorption of mixtures of thermodynamically compatibile and incompatibile polymer pairs from common solvent has been studied in the concentration regime of dilute and semidilute solutions. The critical concentration of overlapping(crossover region) was being dependent on the component ratio estimated from the relationship $[\eta]_{A V}=[\eta]_{A} \varphi_{A}+[\eta]_{B} \varphi_{B}$ Where $[\eta]_{i}$ is intrinsic viscosity of pure components in common solvent, $\varphi_{A}$ and $\varphi_{B}$ are their volume fractions in the mixture and $[\eta]_{A V}$ average intrinsic viscosity of the mixture. It was found that by transition from dilute to semidilute regime the processes of macromolecular aggregation proceed and during adsorption macromolecular aggregares pass onto the adsorbent surface. This transition diminishes in the crossover region. Considering solution of polymer mixture $A+B$ as a solution of $A$ in mixed solvent (solvent $+B$ ) and vice versa, the peculiarities of adsorption are discussed, which are connented with changing thermodynamic qusllity of mixed solvent.

KEY WORDS Adsorption/Mixture / Aggregation/Crossover Region

The present concepts of the properties of polymer solutions suppose the existence of two regimes-those of diluted and semi-diluted solutions[1]. In diluted solution macromolecu-
\end{abstract}


lar coils are separated in space but when reaching critical concentration $C^{x}$ and, respectively, the volume fraction of polymer $\phi^{x}$ the coils begin touching each other and overlapping. Concentration region of coils overlapping (crossover region) corresponds to local concentration inside a coil which is due to concentration of solution. Under these conditions there takes place compressing of coils and decreasing their size. Critical concentration is determined by molecular weight of a polymer and by thermodynamic quality of the solvent. According to various theoretical models $C^{X}$ is inversely proportional to intrinsic viscosity [ $\eta]$, proportionality factor being dependent on the chosen model of coils interaction $[2-6]$

There are also a lot of the data which show that formation of macromolecular associates or aggregates of fluctuation character with long mean lifetime $[7,8]$ is possible in solutions of polymers depending on their concentration and thermodynamic quality of a solvent. Size of aggregates also depends on the polymer molecular weight and solvent nature. Dynamic equilibrium between the aggregated and free macromolecules exists in solutions of any concentration. Proceeding from solutions division into diluted and semi-diluted ones it is evident that the processes of aggregation must begin in the diluted solution and stop when transferring to semi-dilute regime where all the macromolecules overlap and form a network molecular entanglements.

In some our investigations summarized in [9] we have determined conditions of the aggregate formation their sizes and number in the volume unit. A comparison of these results with adsorption data has permitted us to formulate the concept of the aggregative adsorption $[7,9]$. The aggregative adsorption means that in certain concentration region of the solution, where macromolecular aggregates appear both aggregated and nonaggregated macromolecules come to the adsorbent surface under adsorption, the transfer of molecular aggregates being preferable. As a result after adsorption equilibrium being established, the aggregates are practically ab- 
sent in the solution over the adsorbent, but in time their equilibrium concentration is established again.

Aggregative mechanism of adsorption permits explaining some experimental data which exceed the limits of traditional description of adsorption from diluted solutions, $[10,11]$, i.e.: great values of adsorption, which considerably exceed those calculated for monolayers, under simultaneously low fraction of segments bound to the adsorbent surface: inversion of the influence of thermodynamic quality of a solvent on adsorption when changing concentration; dependence of the adsorption value on the ratio adsorbent-solution, etc. [7] The aggregative mechanism of adsorption shows a considerable distinction of polymer adsorption from that of low-molecular substances [7, 9] Under adsorption of polymer molecules not only conformation of the adsorbed molecules but also the size and number of macromolecular aggregates change depending on the solution concentration and thus at any point of the isotherm we meet different structure of adsorbing units and their different ratio in the solution. From this viewpoint it is of interest to consider the adsorption of polymer mixtures from the diluted and semi-diluted solutions in which the conditions of aggregation of each sort molecules should be different. It could be supposed that thermodynamic compatibility or incompatibility of polymers in solution determined by the value of thermodynamic interaction parameter between two polymers would contribute to adsorption of mixturecomponents from common solvent. A number of experimental data on adsorption from mixtures silutions [12-17] has been accumulated till now. As a rule, the selectivity of adsorption of one of the components is observed which was first found in [12-14] It seemed reasonable to suppose that a tendency to segregation of similar macromolecules, i.e. to their aggregation [17] will be to the most extent expressed for the incompatible pairs of polymers. Under these conditions the effect would depend on the total concentration of components in the solution, their ratio and concentration of each component and position of the 
crossover region.

The adsorption of mixtures should be considered from the following positions. Solution of a mixture of two polymers in the common solvent may be described as the solutions of polymer $A$ in the solution of polymer $B$ and at the same time as solution of polymer $B$ in the solution of polymer $A$. In this case thermodynamic quality of the "mixed" solvent (solvent + polymer) for each polymer will differ from the quality of a pure solvent. Adsorption of each polymer will depend on the fact, how the other polymer affects thermodynamic quality of the "mixed" solvent. The adsorption value of each component will be thus determined not only by the affinity of each component to the surface but also by the ratio of components. This simple and self-evident conclusion however leads to great difficulties in the analysis of mixtures adsorption if it will be concerned from classical positions of determination of adsorption isotherms. The first works on adsorption from mixtures dealt with the experimental method to study adsorption from the solution whose concentration remained unchanged only the ratio of dissolved components being changed [12] . Such a method can give information on the preferential adsorption of one or ano ther polymer depending on the presence of the other one. But this method cannot be called a strict one because each change in the components' ratio in solution resulted in the change of thermodynamic quality of a "mixed" solvent for each component, i.e., the adsorption conditions are different for the mixtures with different composition.

The second experimental method is possible where only one component concentration being varied in the mixture solution, the other component concentration being constant. In this case cne can plot the adsorption isotherms for the component with varying concentration. However, such a scheme of the experiment is simply equivalent to the study of the given component adsorption from the solvents of different thermodynamic quality and thus it is difficult to reveal the specificity of mixtures absorption if it exists. 
The third method - corresponds to classical approach to plotting isotherms. It consists in increasing the solution concentration of both components introduced to the solution in the constant ratio. This method also is not strict There will occur simultaneous adsorption of the both components but in different smounts and thus each of them will be dadsorbed from the solvent with permamently changing thermodynamic quality for the given component. Since there are no other possibilities, besides three ones considered, one principal peculiarity in the study of polymer mixtures adsorption follows from this without any experiments. This study cannot be carried out in classical variant of plotting the adsorption isotherms and the very essence of mixtures adsorption itself is determined by the fact that thermodynamic quality of a solvent for each polymer in the mixture proves to be different and changing. Thus, one cannot make "pure" experiment on the adsorption selectivity as it is impossible that a solvent for each polymer possess the same thermodynamic quality as for the other one. The only possibility to make a "pure" experiment is to study the adsorption of components from the melt of two polymers with the absence of any solvent. We have made sucb an investigation [18] but it also was not strictly adsorptional.

Results and Discussion

We have studied the adsorption for some polymer pairs in common solvent. Systems polystyrene-polybutyl-methacrylate (PS-PBMA) and PS-polydimethylsiloxane (PDMS) are thermodynamically incompatible in the solution and a pair polycarbonate (PC) - polycaprolacton (PCL) - compatible in the used solvents.

If one considers the adsorption of mixtures in two regimes of solution (diluted and semi-diluted) it is evident that the conditions of aggregation and adsorption must also depend on the value $\mathrm{C}^{\mathrm{X}}$ for the mixture, i.e., on the region of concentrations where there begins touching and overlapping of coils of both polymers. It is evident that one can- 
not use the value $c^{x}$ for individual components in the solvent since thermodynamic quality of "mixed" solvent in the mixture solution differs from pure solvent and depends on the concentration and ratio of components.

The theory of behaviour of polymer mixtures solutions proceeding from their hydrodynamic properties has not been developed. That is why we propose using the notion of the "averaged" critical concentration for the mixture determined from the "averaged" intrinsic viscosity. Here, in contrast to the approach developed in the work [19-20] we suggest to represent the "averaged" intrinsic viscosity of the mixture $[\eta]_{A V}$ by the ratio: $[\eta]_{A V}=[\eta]_{A} \Psi_{A}+[\eta]_{B} \varphi_{B}$ where $\varphi_{A} \cdot \varphi_{B}=1$ and is a volume fraction of the component in the mixture.

Indeed, for the case of the mixture of macromolecules $A$ and $B$ independent of their thermodynamic compatibility the crossover region will be achieved for each polymer under the concentrations below $C^{x}{ }_{A}$ or $C^{x}{ }_{B}$ because the part of the solution volume is already occupied by the coils of $A$ or $B$ kind. We accept that $C_{A V}^{X}=1 /[\eta]_{A V}$. In this case the average crossover concentration is the function of the ratio of dissolved molecules of the kind $A$ and $B$.

Experimental methods for determining adsorption from mixtures, which we have used, are described in $[16,17]$. Adsorption of mixtures PS-PMBA and PCI-PC was studied under the conditions of constant concentration of mixture in the solution at different ratio of components and adsorption in the system PS-PDMS under constant concentration of one of the components in the system and variable concentration of the second component.

In Fig. 1 the data are presented on the dependence of PDMS adsorption from the mixture with PS in common solvent at different constant concentrations of PS (Above and below $C^{X}$ for PS) in scaled coordinates A (adsorption) $-C / C_{A V}^{X}$. As is evident from the figure in the crossover region and above $\mathrm{C}_{\mathrm{AV}}^{\mathrm{X}}$ a decrease of adsorption is observed after it passes through maximum both for the adsorption of pure 


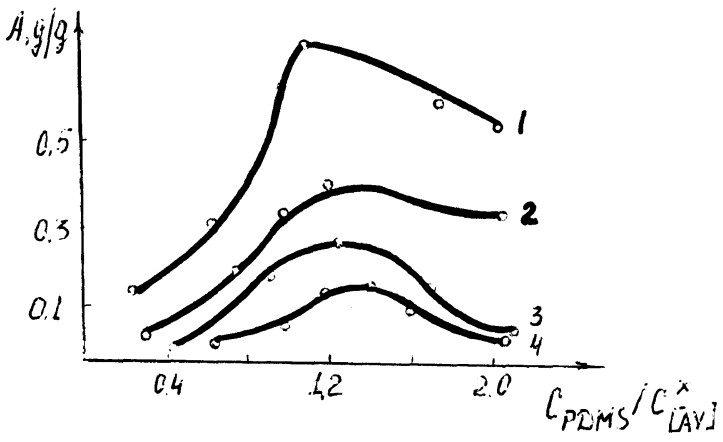

Fig. 1. Dependence of PDMS adsorption from individual solution (I) and its mixtures with PS (2-4) in ethylacetate in scaled coordinates at PS concentration: $2-0.5 ; 3-1.0$; $4-2.5$;

PDMS and from the mixtures containing different amount of PS. Absolute values of adsorption are very high and this corresponds to the mechanism of aggregative adsorption. A decrease of adsorption at concentrations above $C_{\mathrm{AV}}^{\mathrm{X}}$ also meets this mechanism. PS content being increased in the solution (worsening of thermodynamic quality of "mixed" solvent) the adsorption value drops, maximum being kept in the region of scaled concentrations above 1 (above the crossover region). As it was mentioned we connect these regularities with a decrease of solvent quality for PDMS which decreases simultaneously both the sizes of coils and of aggregates.

Data presented in Fig. 2 show that PS adsorbtion in mixture is characterized by smeared maximum in the crossoverregion. Adsorption of PS from mixture is lower and that of PBMA - higher as compared to their adsorption from pure solvent. These data indicate to preferential PBMA adsorption from mixture. In this case it is evident that incompatibility of components promotes preferential adsorption of one of them when adsorbtion of each pure component is approximately the same.

In Fig. 3 data for the considered system are presented otherwise, i.e. In a form of a dependence of the ratio of components adsorption values on the concentration of mixed solvent. PBMA concentration in the solution is plotted on 


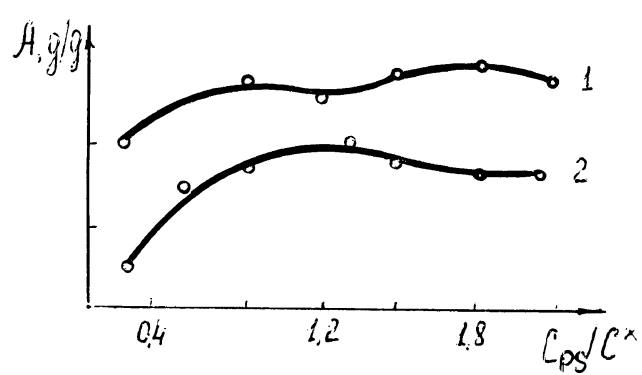

Fig. 2. Dependence of PS adsorption from individual solution (I) and from the mixture with PMBA (2) in carbon tetrachloride in scaled coordinates

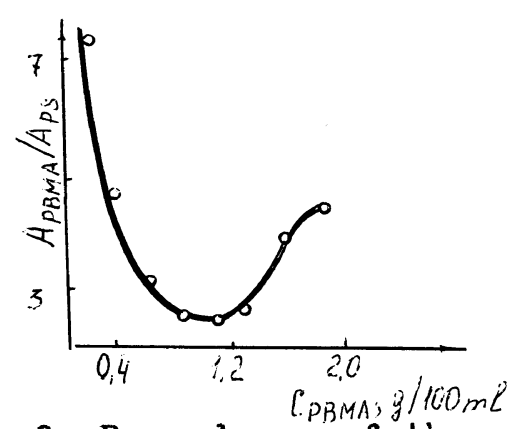

Fig. 3. Dependence of the adsorption ratio A PBMA ${ }^{A}$ PS on PBMA concentration.

the abscissa, total solution concentration $3 \mathrm{~g} / 100 \mathrm{ml}$ being preserved. It is evident from the figure that at any ratio of the mixture components in the solvent PBMA adsorption is preferential. At the same time this dependence is characterized by minimum. A similar minimum (17) was observed in the dependence of the second virial coefficient $A_{2}$ for the ternary system PS - PBMA - solvent. This concentration for the given pair coincides with $\mathrm{C}_{\mathrm{AV}}^{\mathrm{X}}$. After the transition through $C_{A V}^{X}$ the ratio of adsorptions increases again.

For the system PCI - PC $-\mathrm{CHCl}_{3}$ which is compatible in the common solvent, we have studied adsorption from the solution with constant concentration and variable composition. haximum in the crossover region is observed for PCL. Adsorption of PCL is apparently preferential, in spite of low molecular mass and thus PC adsorption begins at its sufficiently high concentrations in the mixture which surpasses $\mathrm{C}_{\mathrm{AV}}^{\mathrm{X}}$. However the adsorption of each component from common solvent is higher than from the mixture, which, as we suppose, is connected with thermodynamic compatibility of components. 


\section{CONCLUSION}

The presented experimental data show that there exists an interrelation between polymer adsorption from mixture, thermodynamic compatibility of components in the solution, change of thermodynamic quality of the "mixed" solvent and critical concentration of the crossover for each component and averaged critical concentration for the mixture. It also proves evident that the aggregation of macromolecules in the mixture solutions, due to the mentioned factors, affects the adsorption in the region of concentrations above and below $\mathrm{C}_{\mathrm{AV}}^{\mathrm{X}}$. As a rule, when passing through $\mathrm{C}_{\mathrm{AV}}^{\mathrm{X}}$ the adsorption value drops.This, among the other reasons, may be due to the fact that the value and number of macromole cular aggregates also depend on $c^{x}$ and are not the same in the region below and above $c^{x}$. As far as formation of aggregates is due to the change of thermodynamic quality of a solvent, it seems evident that the presence of the second component decreases thermodynamic quality of the "mixed" solvent (independent of the fact whether the given pair is compatible in solution or not) and changes the conditions of aggregates formation and their adsorption. High values of adsorption of polymers from pure solvent and from the mixture in common solvent can be explained only from the positions of aggregative mechanism of adsorption. Proceeding from this, differences in behaviour of the studied systems under adsorption in the region below and above the crossover should be connected with the change of aggregation conditions of macromolecules of different nature in both concentratinn regimes and under conditions of continuously changing thermodynamic quality of the solvent.

\section{REFERENCES}

1. P.J. de Gennes. Scaling Concepts in Polymer Physics. Cornel Univ. Press, Ithaca - London, 1979.

2. Jean S, Simionescu B., Neamtu I., Simionescu C. - Polym. Commun. 1986, v. 27, N 4, 113.

3. Nok C., Rudin A. Europ. Polym. J. - 1982, v. 18, N 4, 363. 
4. Bouchaud B., Dacud M. J. Phys. - 1987. v.38, N 11, 1991.

5. Welles D.J. Chem. Soc. Faraday Trans. - 1984, P 1, v. 80, $\mathrm{N} 5,1233$.

6. Ying Q., Chu B. Macromolecules. - 1987. v. 20, N 2, 362.

7. Lipatov Yu.S. Colloid Chemistry of Polymers, Elsevier Science Publ, 1988.

8. Microdomains in Polymer Solutions, Ed. E. Dubin. Plenum. - 1985.

9. Iipatov Yu.S., Sergeeva I. Adv. In Colloid Interface Sci. - 1976, v. 6 .

10. Takahashi A., Kawaguchi M. Adv. Polymer Sci. - 1982, $\mathrm{v} \cdot 46$.

11. Fleer J. In "Reagents in Mineral Technology". - Marcell Dekker Inc. New - York. - 1987, 105.

12. Thies C.J. Phys. Chem. 1966, 70, N 12, 3783.

13. Bothem R., Thies C.J. Coll. and Interface Sci. - 1983, v. $45, \mathrm{~N} 3,512$.

14. Shick M.I., Harvey N.J. Polym. Sci. - Lett. - 1969. B, v. $7, \mathrm{~N} 7$.

15. Lipatov YU.S., Semenovich C. at all. J. Coll. and Interface Sci. - 1982, 986, N 2, 432.

16. Lipatov Yu.S., Sergeeva I., Todosijchuk T., Chornaya V. J. Coll. and Interface Sci. - 1982, 86, B2, 437.

17. Lipatov Yu.S., Todosijchuk T., Chornaya V., Khramova T.J. Coll. and Interface Sci. - 1886, 110, N 1, 1.

18. Lipator Yu.S., Khramova T., Todosijchuk T., Gudova E. J. Coll. and Interface Sci. - 1988, 123, N 1, 143.

19. Pierri E., Dondos A. Europ. Pol. J. - 1987, 23, N 5, 347.

20. Dondos A., Pierri E. Macromol. Chem. - 1988, 189, N 7 , 1685. 